\title{
La exclusión, desigualdad y desarraigo, desde la experiencia de los estudiantes de la Universidad Veracruzana, región Xalapa
}

\author{
Exclusion, inequality and rootlessness, from the experience \\ of students from the Universidad Veracruzana, Xalapa region
}

Jessica Garizurieta Bernabe

\begin{abstract}
RESUMEN
En México existe una escasa pertinencia entre los planes de estudio y las necesidades reales de la sociedad. Debido a esta disparidad, se han suscitado complejas transformaciones dentro del sistema educativo superior. Sin embargo, los resultados obtenidos no han sido óptimos. El presente estudio tiene un carácter exploratorio, con un referente empírico en la universidad pública, trata de visualizar el proceso de exclusión, desigualdad y desarraigo desde la mirada de los estudiantes. Estos escenarios se analizan específicamente a través de la metodología de relatos de vida, contando como plataforma de análisis de los estudiantes de la Universidad Veracruzana, región Xalapa.

Palabras clave: educación superior, exclusión, desigualdad, des-

ABSTRACT

In Mexico there is little relevance between curricula and the real needs of society. Due to this disparity, there have been complex transformations within the higher education system. However, the results obtained have not been optimal. The present study, with an exploratory character and from a university empirical reference of concrete public character, tries to visualize the process of exclusion, inequality and uprooting from the perspective of the students themselves. These scenarios are analyzed specifically through the methodology of life stories, counting through a platform of analysis from students of the Universidad Veracruzana, Xalapa region.

Keywords: higher education, exclusion, inequality, rootlessness, life stories.
\end{abstract} arraigo, relatos de vida. 


\section{INTRODUCCIÓN}

La educación superior se ha considerado fundamental para el desarrollo económico integral y sostenido de una sociedad. Últimamente, esto tiene como base la experiencia de los países llamados "emergentes", cuyas economías se han beneficiado al aumentar la inversión en educación. En este sentido, la educación, además de contribuir al desarrollo económico, podría disminuir el fenómeno de la exclusión en el marco social, económico y cultural (Rodríguez, 2012).

Por otro lado, a través del tiempo, el sistema educativo en México ha buscado soluciones para mejorar los niveles de cobertura, calidad, equidad dentro de ella, pertinencia de los programas de estudio e incrementar la vinculación social; todo mediante diversos programas gubernamentales que han fomentado procesos de planeación, coordinación, programas y evaluaciones. A pesar de este interés, la falta de acceso (exclusión), equidad (desigualdad) y pertinencia (desarraigo) son cuestiones primordiales, sin lugar a dudas interrelacionadas, que han estado presentes dentro del sistema de educación superior. En este sentido, el informe de la Organización para la Cooperación y el Desarrollo Económicos (OCDE, 2013) reporta que en México de los 100 estudiantes que ingresan a la primaria solo 12 terminan el nivel universitario.

Situados en esta perspectiva, este estudio parte de la idea de que el papel de la educación debe estar impregnado de atributos éticos. Para poder visualizar todo esto es necesario que el estudio de este fenómeno social reconozca el carácter multidimensional de la educación. Es importante hacer hincapié en que es muy probable que al impartir la educación surjan impactos negativos, los cuales reciben poca atención por parte de los investigadores porque se considera que la educación en sí misma produce bienestar, lo cataliza. Sin embargo, es importante observar que estas investigaciones son diseñadas en escritorio y por lo tanto alejadas de la realidad de las aulas, impidiendo una asimilación adecuada de la problemática. El proceso educativo está diseñado para hacer creer que se abate la desigualdad e injusticia con el simple hecho de poner la infraestructura al alcance de la sociedad. Sin embargo, es necesario acercarse a los que ponen sus esperanzas en la educación para cuestionarlos sobre si han encontrado, en ese proceso transformador, las respuestas que esperaban.

A partir de lo anterior, este trabajo tiene el objetivo de indagar si la educación superior como proceso de transformación social logra cristalizar dignamente los sueños y esperanzas de los estudiantes y familiares que los apoyan durante todo el

Jessica Garizurieta Bernabe. Profesora de tiempo completo de la Facultad de Contaduría y Administración, región Xalapa, de la Universidad Veracruzana, México. Es doctora en Desarrollo Económico y Sectorial Estratégico y cuenta con especialidad en Administración del Comercio Exterior. Tiene los reconocimientos al perfil ProdeP y del Sistema Nacional de Investigadores. Coordina la Especialización en Administración del Comercio Exterior, de la Universidad Veracruzana, y el Cuerpo Académico "Las Organizaciones en la Sociedad del Conocimiento". Es miembro de la Red de Consejeros del Capítulo PYME-TMEC. Correo electrónico: jgarizurieta@uv.mx. ID: https://orcid.org/0000-0002-1443-4737. 
proceso. Esta investigación presenta la complejidad del sistema de educación superior, planteando que las relaciones que vive un estudiante con las figuras administrativas y académicas son factores importantes que confluyen en la deserción de sus propósitos académicos iniciales; contemplando también cómo el entorno social influye en esta decisión. En este sentido se utilizan dos parámetros para realizar el análisis: a) los aspectos internos: académicos y administrativos y b) los aspectos social, cultural, económico e incluso psicológico que rodean al estudiante y a su familia. Se admite que para conocer lo anterior era necesario entrevistar estudiantes, así las experiencias vividas dentro del complejo proceso de educación superior serían conocidas, al igual que los aspectos que propician relaciones que desembocan en exclusión, desigualdad y desarraigo social, reconociendo que esta complejidad hace que el proceso educativo se torne doloroso y muchas veces se convierta en una experiencia frustrada.

\section{La exclusión, deisgualdad y desarraigo dentro de la educación superior}

El sistema de educación ha sido sometido constantemente a transformaciones, producto del modelo económico neoliberal. Las reformas que podemos ubicar hoy día dentro de la educación superior están asociadas a la emergencia de una vigorosa globalización y privatización del conocimiento, un mercado educativo sin fronteras, nuevos proveedores y, con ellos, nuevos modelos de ofertas educativas (García, 2003), teniendo un sinnúmero de efectos en el sistema educativo, sus instituciones y los actores que en él convergen.

Algunos de los rasgos característicos en materia de educación superior apuntan a "desregular los sistemas, diferenciarlos y diversificarlos en su oferta académica y sus modos de financiamiento e incorporarlos al mundo de la educación globalizada" (Villanueva, 2010, p. 95). No obstante la puesta en marcha de estos cambios ha generado situaciones contradictorias, ya que las políticas establecidas a partir del modelo neoliberal han dejado de tener una función integradora, trayendo como consecuencia un sistema educativo superior que se ha convertido en una fuente de exclusión social.

El concepto de "exclusión social" se remite a la falta de acceso que tienen los ciudadanos a derechos civiles, políticos y sociales, negándoles así un derecho a la participación e igualdad. Esto confiere a la educación un lugar determinante, ya que "no es solamente una condición de acceso a los puestos de trabajo o a las posiciones sociales, es la mayor condición de acceso al ejercicio verdadero de los derechos del ciudadano" (Bourdieu, 1998, p. 102). Sin embargo, la estructura política y económica no facilita el acceso a todos por igual. Siempre existen procesos dolorosos que dificultan a los individuos de estratos sociales vulnerables hacer posible el ejercicio verdadero de los derechos del ciudadano (Bourdieu, 1998).

De esta forma, la exclusión viene determinada por la desigualdad que presentan las personas con respecto a la sociedad en la que viven, que se deriva de la carencia 
a determinados derechos sociales (Hernández, 2008). El concepto de exclusión es multidimensional y multifactorial y, por supuesto, no es un estado sino un proceso que afecta de forma diferente a cada individuo, dependiendo de su potencial y contexto social. En este sentido, las múltiples formas de rechazo tienen un efecto acumulativo, fomentando así la exclusión social. Acorde a la investigación realizada, se puede afirmar que dentro del sistema educativo superior se presentan las siguientes formas de exclusión:

- No poder ingresar a experiencias académicas como la movilidad estudiantil, intercambio, prácticas profesionales y becas. Esta imposibilidad puede estar relacionada a tres aspectos: a) que los requerimientos para el ingreso son altamente rigurosos, b) la incapacidad para cubrir la inscripción y gastos propios de la disciplina en la que se desea incursionar y c) no contar con el tiempo debido a otras exigencias económicas, sociales o culturales, tener alguna discapacidad cognitiva y/o psico-motriz, entre otras.

- No poder acceder a conocimientos y, en consecuencia, al aprendizaje, debido a que: no se cuenta con el recurso necesario, el proceso de enseñanza y aprendizaje no corresponde a las necesidades educativas del estudiante, los materiales didácticos no son los adecuados, o se sufre de discriminación dentro de la institución.

- No contar con el reconocimiento del aprendizaje a través de un título universitario, de forma que no se considera un candidato potencial a desempeñar un trabajo bien remunerado.

Llama la atención cómo diversos procesos contribuyen a alimentar las situaciones de privación social, mucho antes de que el individuo sea parte del sistema educativo superior, puesto que la desventaja educativa se expresa desde la restricción de elección de los estudios que pueden razonablemente estar destinados a una categoría social dada (Bourdieu, 2004), ya que lo que para unos es una elección, para otros es una obligación. Es decir, alguien que decide estudiar música generalmente es por elección, pues está consciente de los gastos que conlleva estudiar una carrera en la que el campo de trabajo es muy limitado; en cambio, hay quienes pese a querer estudiar la carrera que les atrae eligen otra carrera por obligación, pues no cuentan con los recursos necesarios para solventarla, o simplemente su paso por la educación superior es para contar con una herramienta y un título universitario que les garantice un futuro laboral permanente.

Las dificultades estructurales hacen que el sistema escolar mantenga las diferencias sociales preexistentes. Bourdieu, en su libro Capital cultural, escuela y espacio social, hace hincapié en que estos factores, junto con la sobreproducción de programas académicos, han llevado o contribuido a una devaluación de diplomas y posiciones universitarias (Bourdieu, 1998). 
La sociedad occidental y la occidentalizada han permitido que se produzca demasiada estratificación en el acceso a las vías educativas privilegiadas (Illich, Gintis, Greer, Postman, Gross y Fairfield, 1977), a tal grado que la relación directa que se mantiene entre los títulos escolares con los puestos de trabajo ha mermado el objetivo primordial de la educación superior, haciéndola pasar de una formación integral a una mera transmisión de conocimientos técnicos. Aún más, se ha deshumanizado a tal grado que los estudiantes que logran cursar una licenciatura universitaria aceptan como un hecho que el trato injusto es parte de la entrada a la estructura social, aceptando las condiciones establecidas por la sociedad sin considerar el esfuerzo hecho.

Se considera que a partir de la educación superior se puede lograr la igualdad social, puesto que ha sido aceptada universalmente como el mejor camino hacia la obtención de poder, el incremento de la legitimidad personal y la obtención de mayores recursos de enseñanza (Illich et al., 1977), sin embargo, las metas institucionales se contradicen continuamente con los productos institucionales (Illich, 1978).

Es importante hacer hincapié en que "igualdad social" es un término que tiene diferentes significados, dependiendo del contexto en que se mire, ya sea económico, político o educativo. Al mismo tiempo, este término tiende a confundirse con el concepto de "equidad". Por su parte, la equidad significa dar a cada uno lo que merece, es decir, se trata de ser justos en la distribución de las riquezas, mientras que la igualdad se entiende como una cuestión de posibilidad de elección que implica la posibilidad de recibir lo mismo que los demás independientemente de las características individuales y condiciones sociales (Aguado, Pascual, Jiménez-Frías, Sacristán, Ballesteros, Malik y Sánchez, 1999). Esto quiere decir que va a estar determinada por la presencia de igualdad de oportunidades dentro del ámbito que sea estudiado. Bajo este enfoque, la igualdad de oportunidades en el medio educativo parte de la premisa de que la escolarización es un proceso continuo y su medición del logro puede ser vista desde cuatro dimensiones de igualdad (Lozano, 2009, p. 5):

- Igualdad en el acceso, referida al acceso de estudiantes pertenecientes a diversos grupos sociales.

- Igualdad de supervivencia, hace alusión a las posibilidades que tiene un estudiante -independientemente de su grupo social- de permanecer a lo largo de toda la etapa o ciclo escolar, sin abandonar antes de terminar.

- Igualdad de resultados, se considera a la probabilidad de que los aprendizajes y conocimientos son los mismos en un nivel determinado, sin importar el origen social.

- Igualdad en las consecuencias, se refiere a que los estudiantes, independientemente de los resultados, deben tener las mismas oportunidades sociales de acceder al mercado laboral.

Sin embargo en la realidad, como ya se mencionó, en el sistema educativo superior existe una desigualdad de acceso que se traduce en exclusión social, obstaculizando 
muchas veces la supervivencia de los estudiantes en el sistema por falta de recursos financieros.

No obstante, el origen social se considera no solo como el principal determinante de desigualdad sino como la variable más influyente en las cuatro dimensiones de igualdad presentadas y en todos los niveles de experiencia de los estudiantes, pues "el origen de los recursos así como según la naturaleza de la experiencia y los valores asociados a su adquisición, dependen directa y fuertemente del origen social al mismo tiempo que refuerzan su eficacia" (Bourdieu, 2004, p. 27). A partir de este origen es que se define el tipo de vida cotidiano, la capacidad de recursos y el sentimiento de pertenencia a un lugar.

Finalmente, el desarraigo es interpretado como la falta de interés o lazos con el entorno en que se vive, un sentimiento de no-pertenencia con la sociedad en la que se encuentra uno. Es sentir que se está en un lugar, pero que no se pertenece a él, y por consiguiente a ningún lado. En definitiva, todo se reduce a una cuestión entre el individuo y la sociedad; es decir, el círculo que rodea al sujeto y la micro-sociedad en la que se desenvuelve es lo que condiciona la forma en que el individuo percibe la realidad y cómo la asume (Rodríguez, 2012).

El desarraigo social es propiciado en gran parte por la educación, precisamente porque esta centra sus objetivos en la adquisición de conocimientos y desarrollo cognitivo, dejando de lado el plano moral, ético y humano (Rodríguez, 2012). Antes, el sistema escolar aparecía como un lugar al que se iba para aprender cosas universales, era una zona libre para el descubrimiento y el debate de ideas nuevas y viejas, era entonces una comunidad de búsqueda académica (Illich, 1978). Sin embargo, la teoría educativa actual ha ignorado estos principios, trasladando la visión de las instituciones educativas como sitios únicamente de instrucción, y dejando de lado el quehacer cultural y social. Se puede observar cómo, desde la escuela primaria hasta la investigación científica, el trabajo colectivo ya no se induce como antes, al contrario, se tiende a inculcar desde la infancia la idea de la competencia individualista, lo que impulsa a los estudiantes con bagaje cultural occidental escaso a una inclinación pasiva, impidiéndoles crear nuevas formas de integración (Bourdieu, 2004).

Por otra parte, se puede decir que la relación que un individuo mantiene con la escuela, con la cultura que ella transmite y con la lengua que utiliza y exige, depende primordialmente de su medio familiar, del universo escolar y de sus posibilidades para acceder a determinada posición escolar, la que está objetivamente asociada a su grupo de origen (Bourdieu, 2013). Es decir, la forma en que el individuo determina cómo lograr la satisfacción de sus necesidades se ve influenciada por el hogar y la familia, y dichas experiencias se trasladan al medio escolar (Freire, 1972), por lo que, si el individuo debido a su medio familiar no considera la educación como algo primordial o simplemente no cuenta con los recursos necesarios, se verá afectado en su medio 
escolar. En este tenor, muchos de los estudiantes provienen de familias que deciden utilizar sus magros ingresos económicos en satisfacer necesidades urgentes, como ejemplo sería alimentarse en lugar de comprar libros. Asimismo, sentirse parte o no del medio depende de qué tan relacionado se encuentre el estudiante con sus raíces y su cultura. En este sentido, las tradiciones que dan vida a los grupos de clases, unidas con las contradicciones del sistema educativo, suministran las bases y el espacio para la producción del desarraigo social, creando así individuos sometidos, resignados a actos que los llevan a no-reflexionar, desvinculados de su medio social, pues los productos que arroja la enseñanza se encuentran inadaptados a los jóvenes afectados por el mecanismo de lucha de todos contra todos (Bourdieu, 1998).

\section{Marco CONTEXTUAL}

La Universidad Veracruzana (UV) es considerada como una institución líder en el estado de Veracruz, generadora de conocimiento, promotora de cultura y comprometida con el desarrollo del país a través de una visión sustentable.

Su cobertura y área de influencia está planteada a partir de los corredores industriales del estado, de tal manera que la universidad logra ofrecer servicios educativos a lo largo de todo el territorio estatal, a través de cinco regiones universitarias: Coatzacoalcos-Minatitlán, Orizaba-Córdoba, Poza Rica-Tuxpan, Veracruz-Boca del Río y Xalapa. Cada región se encuentra integrada por Facultades, institutos, organismos de difusión de cultura y extensión de los servicios, entre los que se encuentran el Sistema de Enseñanza Abierta, la Escuela para Estudiantes Extranjeros y el Centro de Estudios de Posgrado.

La UV en los últimos años se ha concentrado en una oferta de todo tipo de programas (técnicos, licenciatura y posgrado), que responda a los retos del estado y del país; a fin de incidir de forma significativa en los proceso de desarrollo económico y social, oferta 199 programas académicos de nivel técnico superior, licenciatura y posgrado a través de sus 74 Facultades distribuidas en las cinco regiones. Asimismo ha buscado promover la multiculturalidad, no solo a través de la diversidad cultural de su matrícula, sino también mediante sus casas de estudio y su Universidad Intercultural. Esta última ha desplegado diferentes programas para favorecer el desarrollo de acciones educativas en diferentes niveles educativos (Universidad Veracruzana, 2015) a través de sus cuatro sedes. En cada una oferta la licenciatura en Gestión Intercultural para el Desarrollo, la cual se constituye de cinco orientaciones: Lenguas, Comunicación, Sustentabilidad, Derechos y Salud.

Por otro lado, existen las casas universitarias, creadas como estrategias de vinculación entre el medio rural y el contexto suburbano. Se han creado ocho casas universitarias, denominadas Atlahuilco, Coyopolan, El Conejo, El Paisano, La Chinatla, Molino de San Roque, Tlapala y Vecinos de El Manglar, y su principal objetivo es 
Fortalecer las estrategias de vinculación con las comunidades rurales, indígenas y urbanas, mediante el seguimiento de los Espacios de Casas de la Universidad (Casas UV), la gestión de recursos y la articulación universitaria, para promover la pertinencia académica de la universidad en el marco de la Responsabilidad Social Universitaria [Universidad Veracruzana, 2021, párr. 5].

Específicamente, la región de Xalapa incluye 42 municipios y se encuentra localizada en la porción media del estado de Veracruz, siendo una de las zonas metropolitanas de mayor desarrollo en el estado. En su índice de especialización se observa una fuerte tendencia hacia las actividades primarias, destacando principalmente las actividades económicas dedicadas a los servicios, al comercio y a la administración pública del gobierno del estado y de la propia Universidad Veracruzana.

De las cinco regiones, Xalapa es la que atiende mayormente las necesidades de educación superior, al concentrar el mayor número y diversidad de programas educativos, ya que a través de 32 de las 74 facultades localizadas en esta región se ofertan 61 de los 175 programas a nivel licenciatura con los que la UV cuenta, de los cuales 55 son en modalidad escolarizada, 4 en sistema abierto y 2 en modalidad virtual, siendo mayormente los programas correspondientes al área Económico-administrativa (15), seguido por las áreas Técnica (13) y Humanidades (12). Entre los de menor oferta se encuentran las áreas de Artes (9), Ciencias de la salud (6) y Biológico-agropecuaria (2). No obstante, es importante resaltar que Xalapa es la única región que ofrece programas en el área de artes.

De los 79,804 estudiantes que componían la matrícula total de la UV para el ciclo 2014-2015, ${ }^{1}$ se encuentran matriculados en la región Xalapa 26,570, siendo mayormente dentro del nivel licenciatura $(24,934)$ en su modalidad escolarizada $(90.07 \%)$ (Universidad Veracruzana, 2015). Asimismo, dicha matrícula está representada en $52 \%$ por mujeres y en $48 \%$ por hombres (Universidad Veracruzana, 2015).

Xalapa no solo concentra la mayor cantidad de programas educativos y el mayor número de estudiantes sino también el mayor número de espacios dedicados a la investigación entre institutos, departamentos y unidades. Pese a ser una ciudad basada en actividades del sector terciario, es importante considerar llevar a cabo políticas, dentro de la universidad, enfocadas a impulsar las actividades agropecuarias y forestales, así como de complemento a proyectos productivos que permitan potencializar los municipios rurales por los cuales también está conformada la región.

\section{Metodología}

Para llevar a cabo esta investigación no se puede partir de un diseño preestablecido, tal y como sucede en las investigaciones de corte cuantitativo. El conocimiento que

1 Se establece como referencia el periodo 2014 y 2015 porque el método utilizado, "relatos cruzados", se aplicó en ese lapso de tiempo; es por eso que las cifras corresponden a las mismas fechas. 
se busca como punto de referencia no es primordialmente el avalado científicamente, sino también el de los individuos estudiados, y por consiguiente se mantiene una relación estrecha entre el sujeto y el objeto de conocimiento, de manera que, más allá de una simple evaluación de tipo cualitativo, se busca que las vivencias de los estudiantes cobren valor a través de sus pensamientos, actitudes, opiniones, percepciones, creencias y preferencias. En este sentido, a fin de interpretar y comprender los fenómenos de exclusión, desigualdad y desarraigo social, como producto de los procesos administrativos y académicos de la educación superior, y no solamente de explicarlos, se presenta un acercamiento a la realidad social que viven los jóvenes universitarios a través de la perspectiva denominada "narrativa", aplicada a la percepción que tienen sobre los factores que provocan exclusión, desigualdad y desarraigo en el sistema educativo superior en que ellos están inmersos. Para ello se utilizó la metodología biográfica, específicamente mediante la aplicación del método de "historias de vida" en su forma de método de "relatos cruzados", ya que constituye una reflexión de lo social a partir de historias de varias personas de un mismo entorno, lo que permite explicar a varias voces una misma historia. Este método parte de entrevistas que buscan conocer lo social a través de lo individual, no teniendo que ser este último especial o particular, ya que solo basta con ser parte de una cultura o comunidad que se estudia, de forma tal que la verificación viene dada "a través del contraste de cada narración personal por relación a todas las demás dentro de un mismo medio social" (Pujadas, 1992, p. 56).

El tipo de muestreo es teórico o intencionado, pues lo que se busca es una representatividad cultural, a manera de poder comprender los patrones en torno a los cuales se estructuran los comportamientos y se atribuye sentido a la situación bajo estudio. Por consiguiente y basándonos en las necesidades de la información detectada, y en Creswell, quien dice que "el criterio básico para la selección de los participantes en la investigación cualitativa es que estos provean información necesaria y suficiente para entender y explicar el fenómeno bajo estudio" (Creswell, 2013, citado en Morales, 2015, p. 150), se determinó una muestra no probabilística de 18 estudiantes: tres estudiantes de cada una de las seis áreas académicas de la Universidad Veracruzana: Artes, Biológico-agropecuarias, Ciencias de la salud, Económico-administrativa, Humanidades y Técnica. Para ello, se consideró necesario al menos entrevistar a un hombre y una mujer por área. Es importante hacer hincapié en que se decidió seleccionar números impares por área.

A su vez, se determinó aplicar entrevistas a tres tipos de estudiantes: de nuevo ingreso, de semestres intermedios y de último semestre, los cuales, independientemente de su nivel socioeconómico, cubrieran las siguientes características: estar matriculados dentro de la Universidad Veracruzana, región Xalapa; ser de segundo, 
cuarto u octavo semestre, y pertenecer a algún programa educativo que se enmarque dentro de cualquiera de las seis áreas del conocimiento.

Finalmente, para poder llevar a cabo la recolección de datos se cubrieron tres etapas:

1. En la primera, con el apoyo de los estudiantes, se establecieron los alcances e intenciones de la entrevista, se determinó lo que se quería investigar y por qué, lo que a su vez permitió plasmar algunas inquietudes.

2. La segunda etapa constituyó la realización de la entrevista en profundidad, en la cual no solo se preguntaba de manera global sobre datos referentes a aspectos familiares, sociales y culturales que permitirían explorar y comprender el entorno del individuo, sino también sobre la vida de cada uno de los estudiantes entrevistados, quienes daban detalles de experiencia vivida durante su trayectoria en la universidad.

3. Por último, la tercera etapa se da alrededor de la construcción de los relatos de vida con base en los procesos de análisis e interpretación de los relatos de cada estudiante, lo cual permite a su vez reconstruir las historias, tanto desde un punto de vista particular, al tratar sus vivencias concretas, como social, al permitir comprender procesos colectivos.

De esta forma, como se muestra en la tabla 1, los relatos de vida de cada estudiante permitieron ir pensando, en cada una de las categorías de análisis, la manera de revisar algunos marcos referenciales y orientar la búsqueda en la comprensión de la educación superior y la conformación de la exclusión, desigualdad y desarraigo social, no solo a partir de sus procesos administrativos y académicos.

Tabla 1. Relación de los procesos y factores generadores de exclusión, desigualdad y desarraigo.

\begin{tabular}{llll}
\multicolumn{1}{c}{ Exclusión } & \multicolumn{1}{c}{ Desigualdad } & Desarraigo \\
\hline Procesos administrativos & $\cdot$ Acceso & $\cdot$ Acceso & $\cdot$ Integración \\
& $\cdot$ Integración & $\cdot$ Género & $\cdot$ Contexto social \\
& $\cdot$ Contexto social & $\cdot$ Recursos & $\cdot$ Acceso \\
& $\cdot$ Capacidades cognitivas & & $\cdot$ Integración \\
& y psicomotrices & $\cdot$ Contexto social \\
& $\cdot$ Recursos & $\cdot$ Acceso & $\cdot$ Capacidades cognitivas y \\
\hline Procesos académicos & $\cdot$ Acceso & $\cdot$ Integración & psicomotrices \\
& $\cdot$ Integración & $\cdot$ Contexto social & Ideología \\
& $\cdot$ Contexto social & $\cdot$ Género & Capacidades cognitivas y \\
& y psicomotrices & psicomotrices & Ideología \\
& & $\cdot$ Recursos & \\
\hline
\end{tabular}


A continuación, y a efecto de darle un sentido más amplio a esta investigación, se hace un análisis de los marcos teóricos, contextuales y metodológicos abordados en los párrafos anteriores, a fin de perfilar y definir en un solo análisis las variables que son el fundamento de este trabajo.

\section{LA EXCLUSIÓN}

La institución por sí sola conlleva a exclusiones de ingreso, a partir de las condiciones administrativas impuestas por el modelo educativo integral y flexible (MEIF), el cual tiene el objetivo de fomentar la "formación integral, armónica, intelectual, humana, social y profesional” (Beltrán, 2005, p. 21) a través de:

- La apropiación y desarrollo de valores humanos, sociales, culturales, artísticos, institucionales y ambientales.

- Un pensamiento lógico, crítico y creativo.

- El establecimiento de relaciones interpersonales y de grupo, con tolerancia y respeto a la diversidad cultural.

- Un óptimo desempeño fundado en conocimientos básicos e inclinación y aptitudes para la auto-formación permanente (Beltrán, 2005, p. 22).

Aunque el MEIF tiene buenos fundamentos, la universidad no cuenta con los recursos para operarlo y estos no son suficientes para cubrir las necesidades de los estudiantes.

Los horarios sí convienen, lo que no es el MEIF. Porque si quieres una materia y esta está saturada ya no la puedes meter y si no está saturada no la abren por falta de demanda, aparte de que los horarios quedan todos salteados, no tienes como que el horario todo corrido para aprovechar el tiempo [Magaly, estudiante de quinto semestre de Gestión y Dirección de Negocios].

En nutrición no aplica el MEIF en su totalidad, te dan las materias y no te dejan cambiarlas [Norman, estudiante de sexto semestre de Nutrición].

Lo que no me ha gustado es que se supone que las materias uno las debe escoger y al final te dan lo que quedan de las materias, las sobras. Según... uno escoge el horario [Ricardo, estudiante de último semestre de Biología].

Para la carga de materias todo es en línea, ves las materias y cargas las que quieres, y hay, pero unas están en la mañana, otras en la tarde o no son seguidas [Ángel, estudiante de cuarto semestre de Ingeniería Eléctrica].

Entre las problemáticas que se reconocen se cuentan:

- Oferta restringida en horarios, para las experiencias educativas (EE).

- Mínima flexibilidad en los planes de estudio.

- Dificultad para cursar el plan de estudios en el tiempo mínimo, e incluso en tiempo estándar.

- Dificultad para la movilidad de estudiantes.

- Oferta insuficiente de experiencias educativas. 
- Desconocimiento del plan de estudios y su operación en el MEIF por parte de algunos académicos y tutores.

Hace falta una verdadera flexibilidad, que se le dé apertura a las experiencias educativas que necesitan los estudiantes y con horarios no tan restringidos que, lejos de fomentar una formación integral en los estudiantes, les exigen una permanencia excesiva en la universidad, forzándolos a dejar de lado otras actividades recreativas o de integración social.

Lo malo es la presión de lo que hay que entregar y los horarios... están así como que muy partidos, tienes dos horas clases, después tres horas libres, después una hora clase y, pues no te deja mucho tiempo para hacer otras cosas [Karla, estudiante de cuarto semestre de Biología].

Debido a la carrera no puedes adelantar tareas entre horas porque te llevas como cuatro o cinco horas haciendo una. Y no puedes sacar lo mejor que tienes [Leonardo, estudiante de segundo semestre de Artes Visuales].

Específicamente, los más afectados son aquellos que debido a la falta de recursos se ven en la necesidad de buscar trabajo. Sin embargo, los horarios son una clara limitante.

Por lo mismo de los horarios de la Facultad que luego son tan dispersos dentro del día y en la semana, que es realmente difícil encontrar un trabajo de medio tiempo. Es difícil, y siempre lo que se alcanza a hacer es dar talleres, por horas, en días de la semana y los fines, y eso es lo que me ayuda a sustentarme [Simeón, estudiante de cuarto semestre de Danza Contemporánea].

El proceso es pesado por los horarios, porque, por ejemplo, de mesero te piden que estés ahí ocho horas y me afectaba en clases, no venía, no me daba tiempo de avisarle a los maestros [Hugo, estudiante de cuarto semestre de Artes Visuales].

Intenté trabajar, pero por los horarios se me dificultaba... los horarios en la Facultad son de estar casi todo el día y no hay trabajos de solo unas horas [Adolfo, estudiante de cuatro semestre de Odontología].

Hay entidades en las que se alcanza la flexibilidad de tiempo y los estudiantes cursan sus carreras profesionales en un lapso menor al estándar. Existen planes de estudios en que la conformación no permite más opciones de contenido ni la realización de movilidad; el objetivo es que los estudiantes cursen otras experiencias educativas en espacios y programas diferentes al seleccionado. El primer obstáculo que se presenta en la movilidad es la falta de oferta de espacios en las experiencias educativas, además de las constantes desarticulaciones administrativas presentadas en cada una de las Facultades, que afectan la realización de este trámite, y la infraestructura de la universidad, cuya distancia entre entidades dificulta la movilidad.

No me atrevo a hacer movilidad pues, por lo mismo, que necesito ayuda de alguien para poder moverme y no es tan fácil [Guillermo, estudiante de último semestre de Administración]. 
Hice movilidad con sistema abierto y el proceso fue complicado, no se ponían de acuerdo para la firma de documentos, y te traen de un lado para otro. Por eso no he querido hacerlo de nuevo [Magaly, estudiante de quinto semestre de Gestión y Dirección de Negocios].

En intercambio he visto que hay muchas trabas, al regresar es muy difícil reincorporarse por el papeleo que tienen que hacer, y por el tiempo que requiere no lo hacemos [Rafaela, estudiante de segundo semestre de Arquitectura].

El contexto social de cada estudiante también genera exclusiones, particularmente en los procesos de enseñanza y aprendizaje, como es el caso de los estudiantes $\mathrm{C}$ y $\mathrm{D}$, quienes provienen de otra localidad y tener que viajar constantemente a su hogar hace que se excluyan de ciertas experiencias educativas que puedan surgir durante su estancia en casa.

Ser de fuera ha afectado un poco en la parte académica, más que nada en cuestiones de realizar actividades extraescolares, porque los fines de semana yo viajo a donde soy, entonces ese tiempo no puedo utilizarlo para cuestiones de la escuela [Elizabeth, estudiante de último semestre de Ingeniería Mecánica].

El ser [sic] de fuera ha influido en ocasiones, regularmente viajo cada fin de semana y pues es más pesado que quedarme acá el fin de semana y dedicarme a estudiar y todo eso, como algunos compañeros [Ángel, estudiante de cuarto semestre de Ingeniería Eléctrica].

El proceso de enseñanza-aprendizaje es percibido por los estudiantes como un modelo establecido por el propio docente, siendo este más personal al partir de un proceso de reflexión fundamentado en las necesidades del propio estudiante; de tal manera que aquel que no comprende dicho modelo será excluido y etiquetado por el propio docente, pudiendo ser como alguien trabajador, capaz e inteligente, o por el contrario, como alguien flojo, sin capacidades y falto de conocimientos.

Te tienes que llevar con los maestros correctos para poder ingresar a esos talleres o cursos, y no a todos los que estudian aquí les avisan de los talleres o cursos... me ha tocado ver con mis compañeros que los maestros escogen a sus alumnos favoritos [Hugo, estudiante de cuarto semestre de Artes Visuales].

\section{Desiguladad}

Desde el marco institucional, dentro de la aparente uniformidad de trato en cuestiones de género, aún se logra vislumbrar desigualdades, particularmente en la Facultad de Ingeniería, donde el personal administrativo hace distinción en el trato que da a hombres y mujeres.

Sí he visto la diferencia en cuestión de que, como es un área más de hombres y pocas mujeres, es un poquito diferente la preferencia. Si el maestro o la persona administradora es hombre, el trato es mejor que cuando es una mujer. Si a mí me atiende una mujer es un poco más seco, más duro [Elizabeth, estudiante de último semestre de Ingeniería Mecánica]. 
El trato es parejo, he tenido acceso a todos los servicios, mejor que las niñas. Cuando a la secretaria le toca atenderlas las trata diferente, hasta mal [risa]... [Ángel, estudiante de cuarto semestre de Ingeniería Eléctrica].

Concretamente sobre este último punto, la difusión no es igual en todas las $\mathrm{Fa}$ cultades y se vislumbran desigualdades en la asignación del recurso.

Para lo que es la beca, el proceso de llenar los formatos es un poco tedioso porque no sabes exactamente qué va, hay secciones que son enredadas, y como que la forma de otorgarla no es muy justa, es que no sé cómo tomen algunos criterios, pues hay personas que se ven que lo necesitan y no se la dieron y se la dan a alguien más. Pero como todo es en línea o en papel no sé si eso afecte [Elizabeth, estudiante de último semestre de Ingeniería Mecánica].

$\mathrm{Al}$ analizar esta dimensión desde el punto de vista de tipo y contenido de las relaciones que se establecen en la institución vemos que dentro de los procesos académicos, en su subcategoría de capacidades cognoscitivas y psicomotrices, se destaca como el elemento más relevante la interacción que se establece con los docentes, entiéndanse estos como el cuerpo profesional que actúa, ya sea sobre el colectivo de los estudiantes o en forma individual. De manera general, los estudiantes consideran que un docente tiene capacidad cuando manifiesta un conocimiento amplio sobre el campo de estudio.

Hay maestros que sí saben lo que te enseñan y hay otros que van a enseñarte pero en realidad solo están cubriendo horas y te dan información de relleno [Rafaela, estudiante de segundo semestre de Arquitectura].

Resulta relevante la experiencia que cada estudiante tiene con los distintos docentes, dejando entrever que el buen trato que los estudiantes reciben de sus maestros depende del desarrollo de las habilidades y capacidades cognitivas que tenga el estudiante. Si el estudiante demuestra tener habilidades para la materia en cuestión, el docente le dará un trato preferencial, pero si este no es el caso, y no demuestra manejo de habilidades y conocimientos necesarios en el aula, será rezagado del grupo.

He visto que si te desarrollas más con algún maestro, por ejemplo en campo, ellos se abren más hacia ti que con los que tiene mayor dificultad [Ricardo, estudiante de último semestre de Biología].

Es necesario resaltar que se asignan experiencias académicas vinculadas a profesores que se enfocan a la solidaridad y búsqueda de un aprendizaje integral, sin embargo, también se escuchan anécdotas vinculadas a las desigualdades de trato dentro del aula por parte del docente.

Hay cuestiones distintas que están implícitas en esta dimensión y que tienen que ver con elementos que de alguna manera pertenecen al contexto del estudiante, como sus recursos y su nivel socioeconómico. Si se observan los requerimientos de cada licenciatura presente en la institución educativa, a grandes rasgos no se observarían desigualdades en cuanto a exigencias económicas, sin embargo, una vez adentrados en las disciplinas centrales de cada carrera profesional queda claro que los materiales 
necesarios para el desarrollo de las experiencias educativas en Arquitectura u Odontología no serán los mismos que los requerimientos materiales para una carrera del área Económico-administrativa.

Lo que he visto es que no es una carrera muy cara en comparación con otras, es más lápiz y papel, y lo práctico lo hacemos aquí mismo, es poco el gasto. De las tres ingenierías es la más barata. Por lo que he visto no hay tanto gasto [Ángel, estudiante de cuarto semestre de Ingeniería Eléctrica].

Siento que no he tenido las mismas oportunidades que los demás. Algunos tienen mayores posibilidades de conseguir otros materiales. Entonces, mientras tú trabajas, no sé... con cartón, ellos trabajan con materiales más caros y no les importa tirarlos, mientras que nosotros buscamos reutilizarlos [Laura, estudiante de segundo semestre de Arquitectura].

Si se compara cada una de las carreras universitarias con base en el requerimiento de recursos materiales y/o financieros, encontramos una desigualdad de exigencia que lleva a quienes tienen ingresos altos a contar con mayores posibilidades de permanencia en la carrera profesional que aquellos que no cuentan con los recursos para solventar sus gastos educativos.

Yo siento que las personas que tienen menor ingreso que yo sí les ha costado un poco más en cuestión de poder conseguir para poder ir a un viaje de una visita o más, o para materiales [Elizabeth, estudiante de último semestre de Ingeniería Mecánica].

El costo de los materiales llega a afectarme un poco, porque en mi casa somos tres y los materiales llegan a ser muy caros y tienes que estarte moviendo a las clases cargando todo y si llegas a echarlo a perder ya no puedes presentarlo... me he visto limitada a administrar más mi dinero. Porque, digo... bueno, algo que no compre ahorita que yo quiera, tal vez pueda ayudarme a comprar un material de más calidad y tener un trabajo de más calidad [Rafaela, estudiante de segundo semestre de Arquitectura].

La parte económica sí influye un poco. Hay gastos inesperados y hay que pensar cómo hacerle. Por ejemplo, hay que comprar un bastidor y entonces son... como gastos algo fuertes que no te esperas. Así es que para tener otros ingresos pues... trabajo. El proceso ha sido difícil, no da tiempo y no logro cumplir con las tareas del todo y además, no sé, es un desgaste físico que quieras o no te va a afectar, porque ni siquiera haces las cosas bien, ni pintas bien [Leonardo, estudiante de segundo semestre de Artes Visuales].

Si bien es cierto, que han aumentado los programas destinados a estudiantes de bajos recursos con el fin de asegurar su permanencia y término de carrera universitaria, estos programas no cuentan con un sistema eficiente, que garantice el otorgamiento de becas a estudiantes en situaciones vulnerables. Al mismo tiempo, los montos establecidos en la mayoría de las becas no son altos y llegan a destiempo, lo que lleva a algunos a buscar trabajo a fin de tener una segunda fuente de ingresos. Sin embargo, se comprueba que esta última opción llega a ser de las menos viables, lejos de ayudar al estudiante en el logro de resultados, afecta su desempeño académico.

Es un proceso complicado porque la escuela es de tiempo completo, quizá no de las clases pero, por ejemplo, la mitad del día la ocupo para las clases y la otra mitad para hacer las tareas. Y para 
trabajar es como: "o trabajo o hago las actividades", pero lo que yo hago en la tarde es trabajar y ocupo casi toda la noche para hacer las tareas, y lo poquito que me sobre pues... para dormir. Y eso afecta mi desempeño, porque a veces en las clases me vence el sueño y el desempeño académico ya no es igual, por lo que en ocasiones como que trato de organizarme para obtener mejores resultados en la escuela [Rosa, estudiante tercer semestre de Lengua y Literatura Hispánicas].

\section{Desarraigo}

La universidad ofrece contenidos sociales, políticos y culturales que los estudiantes asimilan e incorporan como resultado de su experiencia a lo largo de su estancia. Estos contenidos forman parte de un marco de experiencias a través de las cuales se trasmiten diversos comportamientos, actitudes y relaciones, mismos que el estudiante incorpora a su propia identidad.

La UV ha avanzado notoriamente en cuanto a la búsqueda de pertenencia e integración social a través de su Universidad Intercultural y de Casas Universitarias. Aún persisten aspectos de desarraigo en los procesos que incurren dentro de la institución-principalmente los académicos-, convirtiendo a la universidad en un espacio donde se expresa la discriminación.

La región de Xalapa se caracteriza por la llegada de estudiantes procedentes de diversos estratos sociales y diversas localidades, que si bien son vistos por la institución como iguales en cuanto a derechos y obligaciones, no lo son por sus pares (demás estudiantes) en cuanto a su origen, ideas, condiciones socioeconómicas y situación familiar, cuestiones que repercuten indiscutiblemente en la manera en que transitan por la universidad y que le imprimen un sello distintivo a su identidad.

Lo que no me ha gustado es que a veces los alumnos excluyen a una persona o hacen sus propios grupos y como que hacen menos a las personas, no todos pero sí algunos [Rosa, estudiante tercer semestre de Lengua y Literatura Hispánicas].

Estas experiencias de discriminación entre pares se encuentran dentro de las aulas, son manifestaciones de diferentes grupos sociales que se crean al interior. Los propios docentes algunas veces generan discriminaciones a partir de los resultados académicos, el origen social o las relaciones intrafamiliares que, al no corresponder con las de la mayoría, lleva a estos estudiantes a sufrir un continuo proceso de etiquetado, con sus particulares consecuencias.

Mis papás me han guiado por un buen camino y me dicen que mi objetivo debe ser mi bienestar.

Hay compañeros que quieren irse de fiesta y, al no hacer lo que ellos, me dicen La Matadita [Zayra, estudiante de sexto semestre de Derecho].

Se observan casos de estudiantes que son discriminados por su religión o su origen, o por demostrar la falta de ciertas habilidades, lo cual deja entrever cómo el propio grupo de pares es el que mayor presión social ejerce de cara a la configuración de las ideologías y los valores sociales. 
En cuanto a las creencias, me he sentido un poco excluida... en religión, eso hace que a veces nos traten diferente. Soy cristiana. Muchas veces hay muchos roces entre los alumnos porque no pienso igual y se tiende a discutir el tema... es más entre compañeros [Laura, estudiante de segundo semestre de Arquitectura].

Los estereotipos sociales y culturales aparecen a lo largo de los relatos, en particular los que se generan dentro de las aulas de la institución educativa, en muchos casos ocasionando choques culturales. Esto quiere decir que, a partir de los grupos sociales conformados en el interior de las aulas, los estereotipos tienden a consolidarse, interiorizándose en los estudiantes a lo largo de su experiencia universitaria.

La universidad influye en los estudiantes, moldeando su identidad. Cada individuo se apropia de un determinado contexto y lo incorpora a su propia vida. En este sentido, la institución sostiene y refuerza el orden social establecido a través de la generación de dos tipos de identidades: la vinculada con el éxito académico, a raíz de calificaciones razonablemente buenas, reconocidas tanto por el estudiante como por el profesor, y la vinculada con el fracaso académico, la cual adoptan aquellos estudiantes cuyas competencias y habilidades no son reconocidas por el docente, llevándolos a una "adaptación silenciosa” al sistema.

Me ha tocado ver con mis compañeros que los maestros escogen a sus alumnos favoritos o se burlan con indirectas [Hugo, estudiante de cuarto semestre de Artes Visuales].

Los juicios y opiniones de los académicos son determinantes en la formación integral del estudiante. Una expresión mal elaborada de un alumno delante del grupo disminuye la autoestima. Los comentarios de un profesor pueden provocar un comportamiento de desarraigo en el estudiante, tanto espacial como social.

\section{Conclusiones}

Tradicionalmente, desde la política pública la educación universitaria ha sido considerada como un factor de igualación de la sociedad y de movilidad, sin embargo, la investigación ha permitido determinar conclusiones semejantes a las formuladas por diversos autores que plantean que el binomio mayor educación universitaria-mayor igualación no es directamente proporcional o de relación lineal (como Bourdieu, Illich, Freire, Giroux, Lozano, Rodríguez Pérez, entre otros). Como se puede identificar a lo largo de la investigación, las condiciones estructurales prevalecientes en esta etapa neoliberal por la que atraviesa la sociedad no han permitido que la educación superior genere situaciones integradoras, por el contrario, es una de las causas por las cuales la educación no alcanza a tener éxito como factor de logros personales y colectivos en México.

Aun cuando las políticas públicas son un factor crucial en el proceso educativo, también lo son las características del entorno que hacen de cada institución un lugar efectivo de aprendizaje para todos los estudiantes. Estos elementos constituyen gran 
parte del logro de la eficacia escolar, por supuesto, ligada a la generación de oportunidades para estudiantes, reconociendo así su derecho a recibir una educación de calidad. De estos componentes del sistema educativo se identificó como los más importantes a los procesos académicos y administrativos internos de cada universidad. Recordando que el estudio se centró en la Universidad Veracruzana región Xalapa, es relevante mencionar que la elección de tal campus fue acertada en varios aspectos, entre ellos los siguientes: no solo concentra la mayor cantidad de programas educativos y el mayor número de estudiantes sino también el mayor número de espacios dedicados a la investigación, tales como institutos, departamentos y unidades.

El análisis sobre la experiencia universitaria de los estudiantes permite reconocer la diversidad cultural que se da en sus aulas, así como la multiplicidad de maneras de conformar los roles que les toca vivir como estudiantes, como jóvenes o como hijos de familia. Ello vino a reforzar empíricamente los estudios y categorías formulados por Pierre Bourdieu sobre el papel del citado capital cultural y hábitos como componentes ligados a la función de la educación como institución de reproducción de desigualdad.

El estudio identifica que el ingreso de los estudiantes al sistema universitario de la UV estuvo marcado por sus expectativas profesionales y personales, no obstante, muchos de los jóvenes coincidieron en percibir una fuerte falta de integración y acceso por parte de la institución, no solo como resultado de la aplicación del modelo educativo MEIF - el cual ha generado una oferta insuficiente de experiencias educativas (EE) y restringida en horarios, dificultad para la movilidad de estudiantes y para cursar el plan de estudios en el tiempo mínimo e incluso en tiempo estándar- sino también por las prácticas tradicionalistas seguidas por algunos docentes. La percepción más fuerte en este sentido fue señalada en un punto excluyente grave: la falta de un reconocimiento de la diversidad cultural y de aprendizaje diferenciado de los estudiantes, teniendo estos últimos que adaptarse a las exigencias del mismo.

En este sentido, se puede afirmar que las transformaciones del sistema universitario deben ser más inclusivas y atender a la diversidad cultural de los estudiantes, mediante programas de atención a la diversidad, relacionados con los sistemas de acceso y de selección, el financiamiento, la concentración de la oferta, la rigidez de los planes de estudio y de la evaluación, y las formas de enseñanza, las cuales cada vez están más centradas en las disciplinas que en los estudiantes.

Se tiene que considerar un cambio profundo en el contrato social que tiene la educación superior en el país, se debe dejar de formar empleados disciplinados y dóciles; para ello es necesaria la reconstrucción del sistema desde un enfoque inclusivo, es decir, es urgente que deje de "prepararlos" para que se adapten a vivir en un mundo injusto, para que acepten esa realidad sin remilgos o cuestionamientos; dando una mayor atención al acceso, la equidad y la pertinencia. 


\section{REFERENCIAS}

Aguado Odina, T., Pascual, J. A., Jiménez-Frías, R. A., Sacristán Lucas, A., Ballesteros Velázquez, B., Malik Liévano, B., y Sánchez García, M. F. (1999). Diversidad cultural e igualdad escolar. Un modelo para el diagnóstico y desarrollo de actuaciones educativas en contextos escolares multiculturales. Revista de Investigación Educativa, 17(2), 471-475. Recuperado de: https:/ / revistas.um.es/rie/article/view/122051.

Beltrán Casanova, J. (2005). El modelo educativo integral y flexible de la Universidad Veracruzana. CPU-e, Revista de Investigación Educativa, 1(1), 1-10. Recuperado de: https://www.redalyc.org/pdf/2831/283121715005. pdf.

Bourdieu, P. (1998). Capital cultural, escuela y espacio social. México: Siglo XXI.

Bourdieu, P. (2004). Los herederos. Los estudiantes y la cultura. México: Siglo XXI.

Bourdieu, P. (2013). En la nobleza del estado: educación de élite y espiritu de cuerpo. México: Siglo XXI.

Freire, P. (1972). Pedagogía del oprimido. México: Siglo XXI.

García Guadilla, C. (2003). Balance de la década de los '90 y reflexiones sobre las nuevas fuerzas de cambio en la educación superior. En M. Mollis (coord.), Las universidades en América Latina: ¿reformadas o alteradas? (pp. 17-37). Argentina: CLACSO.

Hernández Pedreño, M. (2008). Pobreza y exclusión en las sociedades del conocimiento. En M. Hernández Pedreño (coord.), Exclusión socialy desigualdad (pp. 1558). España: Universidad de Murcia.

Illich, I. (1978). La sociedad desescolarizada. Argentina: Ediciones Godot.

Illich, I., Gintis, H., Greer, C., Postman, N., Gross, R., y Fairfield, R. P. (1977). Un mundo sin escuelas. México: Nueva Imagen.

Lozano Medina, M. (2009). Educación superior e igualdad de oportunidades. Tendencias recientes en América latina. Ponen- cia presentada en el X Congreso Nacional de Investigación Educativa. México. Recuperado de: http:/ / www.comie.org.mx/congreso/memoriaelectronica/ v10/pdf/area_tematica_13/ponencias/1233-F.pdf.

Morales Román, G. (2015). Retrato narrativo de las experiencias, retos y motivaciones de la mujer adulta: estudiante del programa doctoral en Educación. Revista Iberoamericana de Educación Superior, 6(16), 143-163. DOI: https://doi.org/10.1016/j.rides.2014.05.001.

OCDE [Organización para la Cooperación y el Desarrollo Económicos] (2013). Programa para la evaluación internacional de alumnos (PIS A). PIS A 2012-Resultados. Recuperado de: http://www.oecd.org/centrodemexico/medios/Mexico\%20Country\%20Note_SPANI SH_final\%20GR1_EGcomments_02_12_2013\%20 final.pdf.

Pujadas Muñoz, J. J. (1992). El método biográfico: el uso de las historias de vida en ciencias sociales. España: Centro de Investigaciones Sociológicas (CIS).

Rodríguez Pérez, M. A. (2012). El desarraigo y la crisis educativa. Revista Cientifica de Farem-Esteli, 1(1), 63-77. Recuperado de: https://rcientificaesteli.unan.edu.ni/ index.php/RCientifica/article/view/468/406.

Universidad Veracruzana (2015). Información estadística institucional. Recuperado de: https://www.uv.mx/ numeralia/matricula/.

Universidad Veracruzana (2015). UV-Intercultural. Recuperado de: http://www.uv.mx/uvi/proyectointercultural/.

Universidad Veracruzana (2021). Casas UV. Recuperado de: https://www.uv.mx/vinculacion/general/casasuv-dgv/.

Villanueva, E. (2010). Perspectivas de la educación superior en América Latina: construyendo futuros. Perfiles Educativos, 32(129), 86-101. Recuperado: http:/ /www. scielo.org.mx/pdf/peredu/v32n129/v32n129a6.pdf.

Cómo citar este articulo:

Garizurieta Bernabe, J. (2021). La exclusión, desigualdad y desarraigo, desde la experiencia de los estudiantes de la Universidad Veracruzana, región Xalapa. IE Revista de Investigación Educativa de la REDIECH, 12, e1242. doi: 10.33010/ ie_rie_rediech.v12i0.1242. 